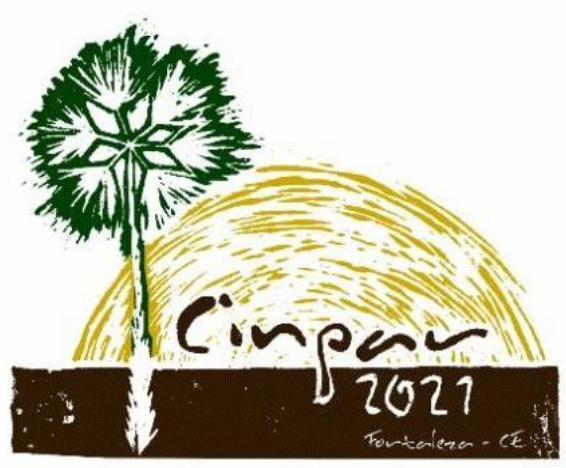

XVII Congresso Internacional sobre Patologia e

Reabilitação das Construções

XVII Congreso Internacional sobre Patología y Rehabilitación de las Construcciones

XVII International Conference on Pathology and Constructions Rehabilitation

FORTALEZA (Brasil), 3 a 5 de junho de 2021

https://doi.org/10.4322/CINPAR.2021.045

\title{
CARACTERIZAÇÃO TÉRMICA DE ARGAMASSAS CIMENTÍCIAS COM DIFERENTES DOSAGENS DE MATERIAIS DE MUDANÇA DE FASE PARA APLICAÇÃO NA CONSTRUÇÃO CIVIL
}

\section{Thermal characterization of cement mortars with different dosages of phase change materials for application in civil construction}

\author{
Adriano Antunes Monteiro', Karoline Teixeira Da Silva², Geovane Santos Borba ${ }^{3}$, Adriane Melo \\ Monteiro $^{4}$ Jorgiane Pires Bezerra ${ }^{5}$, Jessica Maria Bezerra Leite ${ }^{6}$ \\ ${ }^{1}$ École Supérieure d'Ingénieurs des Travaux de la Construction (ESITC Caen), Caen, França, \\ adrianoantunesmonteiro@gmail.com \\ 2 ESITC Caen, Caen, França, karolinetx@gmail.com \\ ${ }^{3}$ Universidade Federal do Ceará, Ceará, Brasil, geosantosb@hotmail.com \\ ${ }^{4}$ Instituto Federal de Ciência, Educação e Tecnologia (IFCE), Ceará, Brasil, driane.mello@gmail.com \\ ${ }^{5}$ IFCE, Ceará, Brasil, jorgianepires@gmail.com \\ ${ }^{6}$ IFCE, Ceará, Brasil, jessicaambl@gmail.com
}

\section{Resumo}

O isolamento térmico de um edifício é essencial para reduzir a perda de energia térmica, em construções situadas em clima temperado, como na França e nos demais países da União Européia. Sua problemática atual é garantir conforto aos usuários e reduzir o impacto energético dos edifícios, impulsionados pela regulamentação francesa cada vez mais exigente e com forte abordagem sustentável. Assim, os materiais de mudança de fase (MCP) incorporados em elementos de vedação prediais são uma alternativa, pois com ele é possível reduzir a necessidade de aquecimento e armazenar energia para melhorar o conforto no verão e no inverno sem usar eletricidade. Esta técnica complementa a absorção de calor pela inércia do edifício. No verão, os MCPs nas paredes absorvem energia do meio ambiente e reduzem o aumento de calor no interior do edifício. No inverno, os MCPs carregam energia de dentro do prédio e a redistribuem no interior à noite, quando a temperatura cai. Assim, este artigo estuda a relação entre a quantidade de material de mudança de fase incorporada em uma argamassa com suas propriedades térmicas. Foram elaborados corpos de provas em laboratório com diferentes dosagens de MCP, seguindo a norma francesa NF EN 196-1 para posterior análise de suas propriedades térmicas. Após o experimento, podemos observar que a condutividade térmica das quatro misturas de argamassa-MCP diminui à medida que a taxa do material de mudança de fase aumenta. A amostra com $20 \%$ de MCP possui a menor condutividade térmica dos corpos de provas analisados, isso significa que o corpo de prova com $20 \%$ de MCP é um isolante melhor. Finalmente, podemos concluir que as argamassas com a adição de MCP ainda apresentam bom desempenho de suas características térmicas, o que nos permite adaptá-las à indústria da construção, reduzindo o custo de eletricidade com conforto térmico.

Palavras-chave: Argamassas Cimentícias. Materiais Sustentáveis. Materiais de mudança de fase.

\footnotetext{
Abstract:

The thermal insulation of a building is essential to reduce the loss of thermal energy, in buildings located in a temperate climate, as in France and other European Union countries. Its current problem is to ensure
} 
comfort for users and reduce the energy impact of buildings, driven by increasingly demanding French regulations and with a strong sustainable approach. Thus, the phase change materials (MCP) incorporated in building sealing elements are an alternative, as it is possible to reduce the need for heating and store energy to improve comfort in summer and winter without using electricity. This technique complements the absorption of heat by the building's inertia. In summer, the MCPs on the walls absorb energy from the environment and reduce heat build-up inside the building. In winter, MCPs carry energy from inside the building and redistribute it inside at night, when the temperature drops. Thus, this article studies the relationship between the amount of phase change material incorporated in a mortar with its thermal properties. Laboratory specimens were prepared with different MCP dosages, following the French standard NF EN 196-1 for further analysis of their thermal properties. After the experiment, we can see that the thermal conductivity of the four mixtures of mortar-MCP decreases as the rate of the phase change material increases. The sample with $20 \% \mathrm{MCP}$ has the lowest thermal conductivity of the analyzed specimens, this means that the specimen with $20 \%$ MCP is a better insulator. Finally, we can conclude that mortars with the addition of MCP still present good performance of their thermal characteristics, which allows us to adapt them to the construction industry, reducing the cost of electricity with thermal comfort.

Keywords: Cement mortars. Sustainable Materials. Phase change materials.

\section{Introdução}

A habitação requer cada vez mais conforto para melhorar a qualidade de vida das pessoas. Em relação ao conforto térmico, os aparelhos elétricos geralmente são utilizados para atender a essa demanda, seja para aquecimento ou refrigeração de ambientes.

A utilização desses aparelhos representam uma grande parte da demanda mundial de energia. A produção de energia gera grandes impactos ambientais para o planeta, principalmente se estiver vinculada à exploração de energias de compostos não renováveis (RAHMOUNI, 2020).

O isolamento térmico de um edifício é essencial para reduzir a perda de energia térmica, em construções situadas em clima temperado, como na França e nos demais países da União Européia. Sua problemática atual é garantir conforto aos usuários e reduzir o impacto energético dos edifícios, impulsionados pela regulamentação francesa cada vez mais exigente e com forte abordagem sustentável (WIRBELDUCOULOMBIER, 2014).

Neste contexto, a última regulamentação térmica em vigor na França, a Regulamentação Térmica (RT) 2012, visava reduzir e limitar as necessidades energéticas em novos edifícios inerentes ao aquecimento, produção de água quente, iluminação, ventilação e ar condicionado (GRENELLE, 2009).

A nova regulamentação ambiental ( $R E$ - Reglamentation environnemental) 2020 visa promover materiais que acumulam calor e o difundem. Desenvolve energias renováveis, gera desenvolvimentos de edifícios inteligentes e edifícios para a produção de energia e a utilização de materiais naturais (FRANCE, 2020).

Nesse sentido, pensando no meio ambiente e na necessidade crescente não só da redução da consumação de energia, mas como na produção de energia, é necessário buscar cada vez mais tecnologias alternativas de fontes de energia e materiais construtivos sustentáveis.

O material de mudança de fase (PCM - Phase Change Materials) é qualquer material capaz de mudar o estado físico dentro de uma faixa de temperatura restrita. Este intervalo é aproximadamente entre $10^{\circ} \mathrm{C}$ e $80^{\circ} \mathrm{C}$. Neste intervalo de temperatura, a mudança de fase predominante permanece fusão / solidificação. Essas temperaturas são acessíveis naturalmente e são onipresentes na vida cotidiana (temperatura ambiente de uma casa, temperatura do corpo humano, água quente sanitária, etc.) (CSTB, [201-]).

Os materiais de mudança de fase incorporados em elementos de vedação prediais são alternativas interessantes, pois com ele é possível reduzir a necessidade de aquecimento e armazenar energia para melhorar o conforto no verão e no inverno (BORDERON, 2012).

Esta técnica complementa a absorção de calor pela inércia do edifício. No verão, os PCMs nas paredes absorvem energia do meio ambiente e reduzem o aumento de calor no interior do edifício. No inverno, os 
PCMs carregam energia de dentro do prédio e a redistribuem no interior à noite, quando a temperatura cai. Assim, para melhor compreesão do comportamento desse material na construção civil, esse artigo tem como objetivo estudar a relação entre a quantidade de material de mudança de fase incorporada em uma argamassa com suas propriedades térmicas.

Desse modo, espera-se saber que porcentagem de PCM adicionado ao material vai obter melhores resultados térmicos.

\section{Metodologia}

O objetivo dos ensaios de laboratório é estudar o impacto da introdução de MCPs numa matriz de argamassa nas propriedades térmicas desta e verificar as suas aplicações na construção. Para isso, foi realizado testes de desempenho térmico nos corpos de prova com diferentes proporções de MCP.

Este estudo foi realizado no Laboratório Comum de Pesquisa da École supérieure d'ingénieurs des travaux de la construction de Caen (ESITC Caen, França), seguindo a norma NF EN 196-1, que descreve o método para determinar a resistência à compressão e a resistência à flexão de uma argamassa de cimento.

O experimento foi dividido basicamente em duas partes principais: a preparação das amostras e a realização dos testes.

\subsection{Preparação das amostras}

Para a preparação das amostras estudadas foi seguido os métodos descritos a seguir:

2.1.1. Fizemos a matriz de concreto convencionalmente, usando as proporções:

- 1 parte de cimento;

- 3 parte de areia;

- $1 / 2$ parte de água.

2.1.2. Foi incorporado o MCP na matriz de concreto inicial usando diferentes proporções:

- Amostra 1: $0 \%$ (não contém MCP)

- Amostra 2: 5\% de MCP

- Amostra 3: 10\% de MCP

- Amostra 4: $20 \%$ de MCP

\subsubsection{Modulação da amostra:}

O concreto enriquecido com MCP foi colocado em uma forma quadrada de poliestireno com dimensões padrão de aproximadamente $210 \mathrm{~mm} \times 185 \mathrm{~mm} \times 6 \mathrm{~mm}$ (Figura 1). A presença do poliestireno torna possível, em primeiro lugar, a cofragem, mas também, graças às suas propriedades térmicas, garantir a boa validade da experiência.

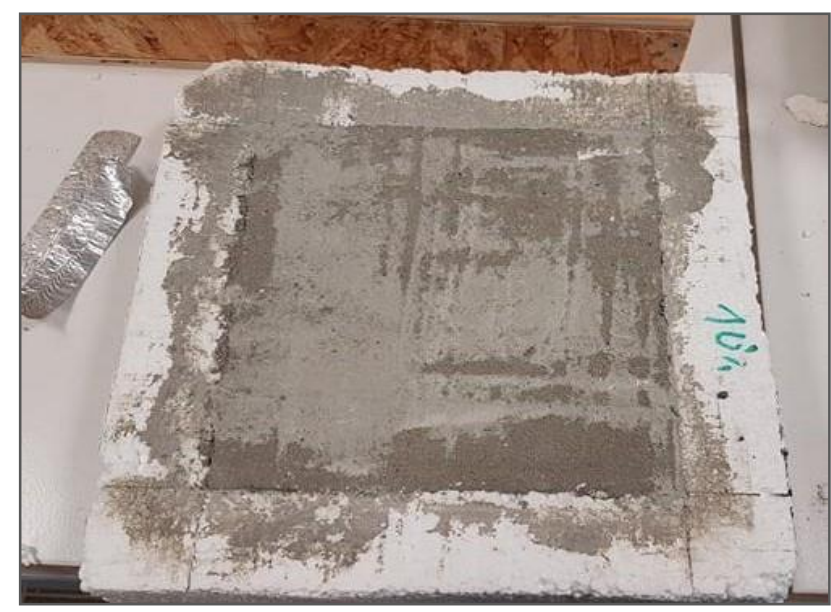


Figure 1 - Amostra estudada

\subsection{Realização dos testes}

Para a análise térmica das amostras foi utilizada a máquina HFM 436 LAMBDA. Este dispositivo mede a condutividade térmica de isoladores. Este parâmetro permite verificar se o material estudado será um bom isolante ou não. Quanto mais baixa for a condutividade, mais isolante é o material.

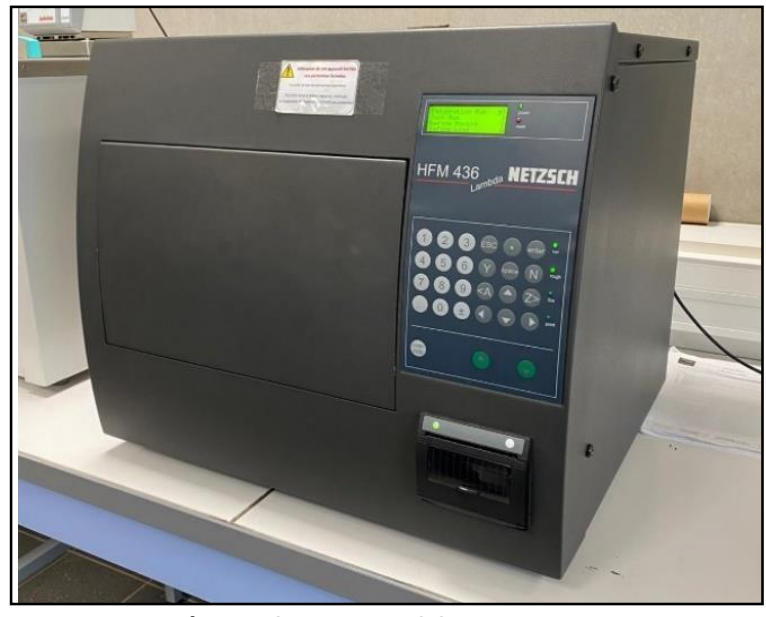

Figure 2 - HFM 436 LAMBDA

Realizamos os testes com a ajuda do manual do usuário HFM 436 LAMBDA. As etapas importantes são as seguintes:

\subsubsection{Configuração da amostra:}

- Fixação de termopares no centro da amostra com fita de alumínio;

- Colocar um tampão no fundo do compartimento onde a amostra é colocada;

- Colocação de amostra usando duas hastes de Teflon.

- Colocação de um tampão na amostra.

- Conexão dos dois termopares.

- Fechamos o compartimento e abaixamos a placa superior para que fique em contato com a amostra.

\subsubsection{Configuração de software:}

- Introduzir as características do projeto e da amostra (massa, dimensões, etc.);

- Escolha das temperaturas da placa superior $\left(30^{\circ} \mathrm{C}\right)$ e inferior $\left(10^{\circ} \mathrm{C}\right)$;

- Definição e aplicação da carga (1,4 MPa);

- Seleção do número de termopares ("todos (valor médio)");

- Seleção dos dois sensores de medição;

- Defina a diferença de temperatura e a temperatura média;

- Salve o método para reutilização com outras amostras;

- Lançamento do julgamento;

- Recuperação de resultados de Excel e Word.

Todos os testes foram feitos seguindo esses métodos, alterando apenas as propriedades da amostra (dimensões, massa, etc.).

\section{Resultados e discussões}

Quatro amostras foram preparadas com diferentes dosagens do material de mudança de fase: sem MCP (0\%), 
$5 \%, 10$ e $20 \%$. Uma vez realizados os experimentos, os resultados para a condutividade térmica e resistência térmica fornecida pelo equipamento HFM 436 LAMBDA foram os seguintes (Tabela 1):

\begin{tabular}{|c|c|c|c|c|c|c|c|c|}
\hline Amostra & $\begin{array}{c}\text { Temp. } \\
\text { Média } \\
{ }^{\circ} \mathbf{C}\end{array}$ & $\begin{array}{c}\text { Delta } \\
\text { Temp. } \\
\mathbf{K}\end{array}$ & $\begin{array}{c}\text { Flux. } \\
\text { de calor } \\
\mathbf{W} / \mathbf{m}^{2}\end{array}$ & $\begin{array}{c}\text { Condutividade } \\
\text { térmica } \\
\mathbf{W} /(\mathbf{m} \cdot \mathbf{K})\end{array}$ & $\begin{array}{c}\text { Resistência } \\
\text { térmica } \\
\left(\mathbf{m}^{\mathbf{2}} \cdot \mathbf{K}\right) / \mathbf{W}\end{array}$ & $\begin{array}{c}\text { Gradiente } \\
\mathbf{d e ~ T e m p .} \\
\mathbf{K} / \mathbf{m}\end{array}$ & $\begin{array}{c}\text { Duração } \\
\text { hh:mm:ss }\end{array}$ & $\begin{array}{c}\text { Pressão de } \\
\text { carga } \\
\mathbf{k P a}\end{array}$ \\
\hline $0 \%$ & 17.8 & 7.4 & 240.18 & 1.67866 & 0.03098 & 143.0 & $00: 51: 36$ & 1.7 \\
\hline $5 \%$ & 22.0 & 4.4 & 120.63 & 1.44196 & 0.03606 & 83.7 & $00: 36: 28$ & 1.4 \\
\hline $10 \%$ & 19.6 & 8.3 & 211.42 & 1.29286 & 0.03945 & 163.6 & $00: 42: 46$ & 1.4 \\
\hline $20 \%$ & 21.4 & 7.9 & 199.75 & 1.29441 & 0.03940 & 154.2 & $00: 40: 49$ & 1.6 \\
\hline
\end{tabular}

Tabela 1 - Resultados dos testes

Desta forma, para uma melhor visualização do comportamento térmico das argamassas em função da variação da taxa de mudança de fase do material, foi gerado o seguinte gráfico.

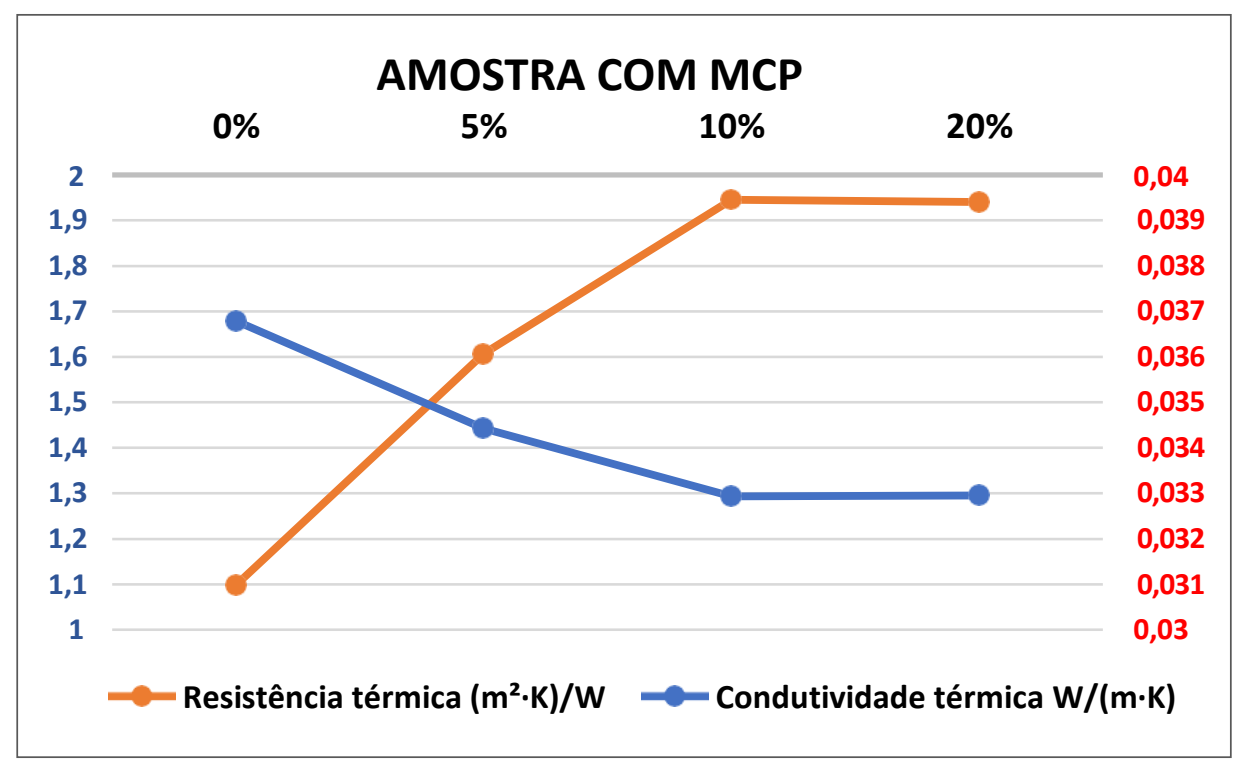

Figura 3 - Comportamento térmico das amostras

Podemos notar que a condutividade térmica das quatro diferentes misturas de argamassa MCP diminui à medida que a taxa de material mudança de fase do material aumenta, ou seja, a resistência térmica da argamassa é diretamente proporcional à taxa de MCP contida na matriz.

Isso quer dizer que, se introduzido no produto cimenticio de vedação durante a construção de uma parede, o MCP pode armazenar energia cinética durante um período do dia e liberar no outro, ajudando assim a equilibrar a temperatura do ambiente. Logo, com ele é possível reduzir a necessidade de utilização de equipamentos para controle de temperatura, como aparelhos de ar condicionado e aquecedores, para manter o conforto térmico (BORDERON, 2012).

Desse modo, a utilização do MCP irá auxiliar a reduzir a consumação de energia elétrica e, consequentemente, diminuir com os impactos ambientais causados pela atividade de produção energética, 0 que se encaixa no que a RE 2020 tem como objetivo, para a promoção do desenvolvimento sustentável (FRANCE, 2020).

Fazendo uma comparação entre os resultados obtidos, observou-se que a amostra com $20 \%$ de MCP apresenta a menor condutividade térmica do concreto MCP. Isso significa que a amostra com $20 \%$ de MCP é um isolante melhor do que as amostras com $10 \%, 5 \%$ e $0 \%$. 
Entretanto, é importante pontuar que a diferença na resistência térmica entre a amostra de $10 \%$ e $20 \%$ é muito pequena, o que cabe a possibilidade de realização de um estudo mais aprofundado para saber se em termos gerais seria mais viável a utilização do MCP à $10 \%$.

\section{Considerações finais}

A realização deste procedimento em laboratório permitiu analisar o comportamento térmico de argamassas com diferentes níveis de material de mudança de fase na sua composição.

A quantificação do material de mudança de fase é muito importante porque a seleção do material torna a escolha mais objetiva para obter o maior benefício com o mínimo de material.

Conclue-se que as propriedades térmicas da argamassa são melhores quando incorporada em sua composição, ou seja, quando $20 \%$ do material de mudança de fase é adicionado à argamassa, há menor condutividade térmica e maior resistência térmica.

Por fim, pode-se concluir que as argamassas com adição de MCP apresentam sempre um bom desempenho térmico, o que nos permite adaptá-las à indústria da construção com maior facilidade e segurança, reduzindo assim o custo da eletricidade para o conforto térmico.

No entanto, além do desempenho térmico, devemos questionar as propriedades mecânicas do concreto enriquecido com MPC para poder utilizá-las na construção civil. Além disso, se seria mais viável a utilização à $10 \%$ visto que a diferença entre as amostras de $10 \%$ e $20 \%$ é muito pequena.

\section{Referências Bibliográficas}

Borderon, J. (2012). Intégration des matériaux a changement de phase comme système de régulation dynamique en rénovation thermique. Thèse de doctorat. Université de Lyon, ENTPE. 2012. Disponible dans : https://hal.archives-ouvertes.fr/tel-01614879/document.

CSTB. (201-). Quelles évolutions techniques ? Liquides ou solides - les matériaux à changement de phase. [201-]. Disponible dans: http://www.cstb.fr/archives/webzines/editions/decembre-2006/quellesevolutions-technologiques.html

Rahmouni, S. (2020). Evaluation et Amélioration Energétiques de Bâtiments dans le cadre du Programme National d'Efficacité Energétique. [Thèse de Doctorat, Université Mostapha Ben Boulaid de Batna 2]. Thèses en ligne de l'Université Batna $2:$ http://eprints. univbatna2.dz/1830/1/Th\%C3\%A8se\%20Rahmouni.pdf.

Wirbel- Ducoulombier, L. (2014). Conception d'un nouveau système d'isolation par l'extérieur pour le bâtiment. Génie civil. École Centrale de Lille, 2014. Français. NNT : 2014ECLI0013. tel-02372099.

Grenelle. (2009). LOI n 2009-967 du 3 août 2009. Programmation relative à la mise en œuvre du Grenelle de l'environnement (1).

France. (2020). Réglementation Environnementale 2020. Loi pour l'évolution du logement, de l'aménagement et du numérique (ELAN). Loi promulguée : 27. Nov. 2018. 